PROCEEDINGS OF THE

AMERICAN MATHEMATICAL SOCIETY

Volume 125, Number 6, June 1997, Pages 1823-1830

S 0002-9939(97)04026-4

\title{
PRODUCTS WITH LINEAR AND COUNTABLE TYPE FACTORS
}

\author{
S. PURISCH AND M. E. RUDIN
}

(Communicated by Franklin D. Tall)

\begin{abstract}
The basic theorem presented shows that the product of a linearly ordered space and a countable (regular) space is normal. We prove that the countable space can be replaced by any of a rather large class of countably tight spaces. Examples are given to prove that monotone normality cannot replace linearly ordered in the base theorem. However, it is shown that the product of a monotonically normal space and a monotonically normal countable space is normal.
\end{abstract}

\section{INTRODUCTION}

By a space we mean a Hausdorff, regular topological space.

The origin of this paper was a theorem of S. Purisch that the product of a space embeddable in a linearly ordered (called suborderable) space and a countable space is normal. A number of possible generalizations arose.

The proof itself can be generalized to replace the countable space by any space in the class $\mathcal{N}$ of countably tight spaces whose product with all paracompact suborderable spaces is normal. For instance a countably tight $\sigma$-compact space would do, or a countably tight, paracompact, $\sigma$-locally compact space. This latter generalization of the original proof was independently observed by Peter Nyikos. To see that such a space is in $\mathcal{N}$ recall:

Lemma $1.1([\mathrm{M}])$. The product of a paracompact space and a $\sigma$-locally compact, paracompact space is paracompact.

In Theorem 1 we prove that the product of a suborderable space with any space from class $\mathcal{N}$ is normal. Linearly ordered spaces are monotonically normal and monotonically normal spaces have many of the properties of linearly ordered spaces. However, Theorem 2 provides a monotonically normal space and a countable space whose product is not normal. Nonetheless, Theorem 3 gives a proof that the product of a monotonically normal space with a monotonically normal countable space is always normal.

When referring to a suborderable space we will assume a fixed linear order compatible with its topology.

Received by the editors August 14, 1995.

1991 Mathematics Subject Classification. Primary 54D18, 54B10, 54D15, 54A25.

Key words and phrases. Linearly ordered, countable, monotonically normal, product.

(c)1997 American Mathematical Society 
Theorem 1. The product of a suborderable space with a countably tight space whose product with all paracompact suborderable spaces is normal, is itself normal.

Let $X$ be a suborderable space. A left gap in $X$ is a nonempty clopen initial segment in $X$ that has no maximum. A left gap is a left $Q$-gap if there is no order preserving copy of a stationary set (i.e., an order preserving homeomorphism onto a stationary subset of an uncountable regular cardinal with the order topology) in the gap which is cofinal in the gap. (For example, see $[\mathrm{P}]$.) A right gap and right $Q$-gap are defined analogously. In $[\mathrm{GH}]$ it is shown that a linearly ordered space is paracompact if and only if all of its gaps are $Q$-gaps.

The greatest ordered compactification ([F]) $b X$ of $X$ is the ordered compactification of $X$ whose order extends the order of $X$ and whose growth is order-isomorphic to the set of gaps of $X$. Hence, to each left (right) gap $A$ in $X$ corresponds the gap point $p_{A}$ in $(b X-X)$, which is the sup (inf) in $b X$ of $A$.

Let $X^{*}$ denote $X \cup\{p \in(b X-X) \mid p$ is a non-Q-gap point of $X\}$ and let $\leq$ denote the order relation on both $X$ and $X^{*}$.

In $[\mathrm{P}]$ it is shown that $X^{*}$ is paracompact. Note that if $p \in\left(X^{*}-X\right)$ is a left gap point, then $\left\{a \in X^{*} \mid a \leq p\right\}$ is clopen in $X^{*}$. If $p$ is a right gap point, then $\left\{a \in X^{*} \mid p \leq a\right\}$ is clopen in $X^{*}$.

Through Lemma 1.4 let $Y$ be a countably tight normal space and $C$ a closed subset of $(X \times Y)$ contained in an open subset $O$. Let $C^{\prime}$ be the closure of $C$ in $\left(X^{*} \times Y\right)$.

Interval notation refers to intervals of $X$.

Lemma 1.2. Let $p \in\left(X^{*}-X\right)$; say $p$ is a left non-Q-gap point where $A_{p}=$ $(-\infty, p)=\{x \in X \mid x<p\}$. If $\left\langle p, y_{0}\right\rangle \in C^{\prime}$ for some $y_{0} \in Y$, then $\pi_{X}\left(C \cap\left(A_{p} \times\left\{y_{0}\right\}\right)\right)$ is cofinal in the non-Q-gap $A_{p}$, where $\pi_{X}:(X \times Y) \rightarrow X$ is the projection map.

Proof. Let $\left\langle p, y_{0}\right\rangle \in C^{\prime}$. Assume $\pi_{X}\left(C \cap\left(A_{p} \times\left\{y_{0}\right\}\right)\right)$ is not cofinal in $A_{p}$. Then there exists $x_{0} \in A_{p}$ such that $C \cap\left(\left(x_{0}, p\right) \times\left\{y_{0}\right\}\right)=\emptyset$. (Recall that $\left(x_{0}, p\right)=\{x \in$ $\left.X \mid x_{0}<x<p\right\}$.)

Now, by induction, we construct an increasing sequence $\left\{x_{\alpha} \mid \alpha<\kappa\right\}$ cofinal in $A_{p}$ for some ordinal $\kappa$ as follows.

For $0<\beta$ assume $\left\{x_{\alpha} \mid \alpha<\beta\right\}$ has been chosen. If $\left\{x_{\alpha} \mid \alpha<\beta\right\}$ is cofinal in $A_{p}$, define $\kappa=\beta$ and cease the construction.

Otherwise, for limit $\beta$ just choose $x_{\beta} \in A_{p}$ with $x_{\beta}>x_{\alpha}$ for all $\alpha<\beta$.

If $\beta$ is a nonlimit ordinal, $\beta=\alpha+1$ for some $\alpha<\beta$. Let $C_{\alpha}=C \cap\left(\left(x_{\alpha}, p\right) \times Y\right)$. Then $\left\langle p, y_{0}\right\rangle \in C^{\prime}$ implies $y_{0} \in \overline{\pi_{Y}\left(C_{\alpha}\right)}$. So, by the countable tightness of $Y$, there exists a countable $Y_{\alpha} \subset \pi_{Y}\left(C_{\alpha}\right)$ such that $y_{0} \in \overline{Y_{\alpha}}$. Since $Y_{\alpha}$ is countable and $A_{p}$ is a non- $Q$-gap and therefore does not have countable cofinality, we can choose $x_{\alpha+1} \in A_{p}$ such that for every $y \in Y_{\alpha}$ there is an $x \in\left(x_{\alpha}, x_{\alpha+1}\right)$ with $\langle x, y\rangle \in C_{\alpha}$.

Since $A_{p}$ is a non- $Q$-gap, $\left\{x_{\alpha} \mid \alpha<\kappa\right\}$ has a cluster point $q \in A_{p}$. Since $q \in\left(x_{0}, p\right)$, by our definition of $x_{0},\left\langle q, y_{0}\right\rangle \notin C$. So $\left\langle q, y_{0}\right\rangle$ has a basic neighborhood $(U \times V)$ in $(X \times Y)$ missing $C$. However, there is some $\alpha<\kappa$ with $\left(x_{\alpha}, x_{\alpha+1}\right) \subset U$. Since $y_{0} \in \overline{Y_{\alpha}}$, there is $y \in Y_{\alpha} \cap V$, and, by our choice of $x_{\alpha+1}$, there is $x \in\left(x_{\alpha}, x_{\alpha+1}\right)$ with $\langle x, y\rangle \in C$. But $\langle x, y\rangle \in C \cap(U \times V)$ is a contradiction to our choice of $(U \times V)$ and Lemma 1.2 is proved.

Lemma 1.3. Let $D=(X \times Y)-0$. Then $C^{\prime} \cap D^{\prime}=\emptyset$.

Proof. Recall that $O$ is an open set in $(X \times Y)$ containing $C$. Observe that $C \cap D=$ $\emptyset$. 
Let $p \in X^{*}-X$, say $p$ is a left non- $Q$-gap point. Let $\left\langle p, y_{0}\right\rangle \in C^{\prime}$ for some $y_{0} \in Y_{0}$. By Lemma 1.2, $\pi_{X}\left(C \cap\left(A_{p} \times\left\{y_{0}\right\}\right)\right)$ is cofinal (as well as closed) in $A_{p}$. If $\left\langle p, y_{0}\right\rangle \in D^{\prime}$, then, replacing $C$ by $D$ in Lemma $1.2, \pi_{X}\left(D \cap\left(A_{p} \times\left\{y_{0}\right\}\right)\right)$ is cofinal and closed in $A_{p}$. Since $A_{p}$ is a non- $Q$-gap there are no two disjoint closed cofinal subsets of $A_{p}$. Thus $\left\langle p, y_{0}\right\rangle \notin D^{\prime}$. That is, there is an $x \in A_{p}$ and an open neighborhood $V$ of $y_{0}$ in $Y$ such that $((x, p) \times V) \subset O$, as required.

Lemma 1.4. Assume that $Y$ is in class $\mathcal{N}$. That is, $Y$ is countably tight and the product of $Y$ with any paracompact suborderable space is normal. Then there is an open $U$ in $(X \times Y)$ such that $U \subset \bar{U} \subset O$.

Proof. Let $O^{\prime}=\bigcup\left\{R\right.$ open in $\left.\left(X^{*} \times Y\right) \mid R \cap(X \times Y) \subset O\right\}$. Obviously $O^{\prime}$ is open in $\left(X^{*} \times Y\right)$ and $O^{\prime} \cap(X \times Y)=O$.

To see that $C^{\prime} \subset O^{\prime}$, recall that $C \subset O$ and, for $\langle p, y\rangle \in\left(C^{\prime}-C\right), p \in X^{*}-X$. Again assuming that $p$ is a left non- $Q$-gap point, by Lemma 1.3, for some $x \in A_{p}$ and open neighborhood $V$ of $y$ where $\langle p, y\rangle \in C^{\prime},((x, p) \times V) \subset O$. It follows that $\langle p, y\rangle \in O^{\prime}$.

Since $X^{*}$ is a paracompact suborderable space $([\mathrm{P}])$, by our choice of $Y,\left(X^{*} \times Y\right)$ is normal. Therefore, there is an open $W$ in $\left(X^{*} \times Y\right)$ such that $C^{\prime} \subset W \subset \bar{W} \subset O^{\prime}$ where $\bar{W}$ is the closure of $W$ in $\left(X^{*} \times Y\right)$. Hence

$$
\begin{aligned}
C & =\left(C^{\prime} \cap(X \times Y)\right) \subset(W \cap(X \times Y)) \\
& =U \subset \bar{U} \subset(\bar{W} \cap(X \times Y)) \subset\left(O^{\prime} \cap(X \times Y)=O,\right.
\end{aligned}
$$

as desired.

Theorem 2. There is a monotonically normal space $X$ and a countable (regular Hausdorff) space $Y$ such that $(X \times Y)$ is not normal.

Proof. We begin with some elementary but rather messy combinatorics on subsets of $\omega$ which make it possible to define our topologies.

We inductively choose for each $f \in 2^{<\omega}$ an infinite subset $M_{f}$ of $\omega$ as follows:

For $f \in 2^{0}$, define $M_{f}=\omega$.

If $n \in \omega, f \in 2^{n}, M_{f}$ has been chosen, and $g$ and $g^{\prime}$ are the two terms of $2^{n+1}$ extending $f$, then choose $M_{g}$ and $M_{g^{\prime}}$ to be two infinite, disjoint sets whose union is $M_{f}$ which separate the two smallest members of $M_{f}$.

Let $\mathcal{M}=\left\{M_{f} \mid f \in 2^{<\omega}\right\}$.

For $i \in \omega$, let $\mathcal{M}_{i}=\{M \in \mathcal{M} \mid i \in M\}$.

By induction choose $f_{n} \in 2^{n}$ for each $n \in \omega$ so that, for $n>0, f_{n}$ extends $f_{n-1}$ and $M_{f_{n}}$ does not contain the minimal element in $M_{f_{n-1}}$. Let $\mathcal{M}_{\omega}=\left\{M_{f_{n}} \mid n \in \omega\right\}$.

Let $\mathcal{A}$ be the set of all nonempty finite subsets of $\omega_{1}$.

We next define for each $\alpha \in \omega_{1}$, disjoint infinite sets $I_{\alpha 0}$ and $I_{\alpha 1}$ whose union is $\omega$ such that for all disjoint $A$ and $B$ in $\mathcal{A}$ and $M \in \mathcal{M},\left[\left(\bigcap_{\alpha \in A} I_{\alpha 0}\right) \cap\left(\bigcap_{\alpha \in B} I_{\alpha 1}\right) \cap M\right]$ is infinite.

We prove there are such sets by induction on $\omega_{1}$.

If $\beta=0$, for each $n \in \omega$ and $f \in 2^{n}$ choose distinct $i_{f 0}$ and $i_{f 1}$ from $M_{f}-\{i \in \omega \mid i$ is $i_{g 0}$ or $i_{g 1}$ for some $\left.g \in 2^{<n}\right\}$. Since the $M_{f}$ 's are disjoint for $f$ 's in $2^{n}$ for a fixed $n$, the chosen points at this $n$ are all distinct. Define $I_{\beta 0}=\left\{i_{f 0} \mid f \in 2^{<\omega}\right\}$ and $I_{\beta 1}=\left(\omega-I_{\beta 0}\right)$. Since $\left\{i_{f 1} \mid f \in 2^{<\omega}\right\} \subset I_{\beta 1}$, both sets intersect every $M \in \mathcal{M}$ in an infinite set.

Assume $\beta \in \omega_{1}, \beta \neq 0$, and $I_{\alpha 0}$ and $I_{\alpha 1}$ have been defined for all $\alpha<\beta$ having all the desired properties for $A$ and $B$ contained in $\beta$. Index $\beta=\left\{\alpha_{n} \mid n \in \omega\right\}$. 
Then, as in the case for $\beta=0$, by induction for each $n \in \omega$ and $f \in 2^{n}$ choose distinct $i_{f 0}$ and $i_{f 1}$ from:

$$
\left[\left(\bigcap_{m<n} I_{\alpha_{m} f(m)}\right) \cap M_{f}\right]-\left\{i \in \omega \mid i \quad \text { is } \quad i_{g 0} \quad \text { or } \quad i_{g 1} \quad \text { for some } g \in 2^{<n}\right\} .
$$

Since the first set is infinite by hypothesis and the second is finite because $2^{<n}$ is finite, we have no trouble choosing two distinct elements from this set. Define $I_{\beta 0}=\left\{i_{f 0} \mid f \in 2^{<\omega}\right\}$ and $I_{\beta 1}=\left(\omega-I_{\beta 0}\right)$. Again the fact that $\left\{i_{f 1} \mid f \in 2^{<\omega}\right\} \subset I_{\beta 1}$ ensures the desired intersection property for our induction hypothesis.

For each $A \in \mathcal{A}$ define $I_{A 0}=\bigcap_{\alpha \in A} I_{\alpha 0}$ and $I_{A 1}=\bigcap_{\alpha \in A} I_{\alpha 1}$.

For $\alpha \in \omega_{1}$, define $\mathcal{A}_{\alpha}=\left\{A \in \mathcal{A} \mid A \subset\left(\omega_{1}-\alpha\right)\right\}$.

We now define our spaces $X$ and $Y$.

Let $X=\omega_{1} \times(\omega+1)$ topologized as follows:

(1) Every singleton from $\omega_{1} \times \omega$ is a basic open set in $X$.

(2) For $\alpha<\omega_{1}, \lambda<\alpha$ (where $\lambda=-1$ is allowed to get something less than $0), M \in \mathcal{M}_{\omega}$, and $A \in \mathcal{A}_{\alpha}$, a basic open set (for $\left.\langle\alpha, \omega\rangle\right)$ is $U_{M A \lambda}(\alpha)=$ $\{\langle\beta, \omega\rangle \in X \mid \lambda<\beta \leq \alpha\} \cup\left\{\langle\beta, i\rangle \in X \mid \lambda<\beta \leq \alpha\right.$ and $\left.i \in\left(I_{A 0} \cap M\right)\right\}=$ $(\lambda, \alpha] \times\left(\left(I_{A 0} \cap M\right) \cup\{\omega\}\right)$.

Two members of $\mathcal{M}_{\omega}$ intersect in a member of $\mathcal{M}_{\omega}$ and $I_{A 0} \cap I_{B 0}=I_{(A \cup B) 0}$. If $\lambda<\alpha \leq \beta<\omega_{1}, A \in \mathcal{A}_{\alpha}$, and $B \in \mathcal{A}_{\beta}$, then $(A \cup B) \in \mathcal{A}_{\alpha}$ and $U_{M A \lambda}(\alpha) \cap$ $U_{M B \lambda}(\beta)=U_{M(A \cup B) \lambda}(\alpha)$. The intersection of two "basic open sets" is clearly another one, so this is indeed a topology. For each distinct $i \in \omega$ and $j \leq \omega$, there are disjoint $M \in \mathcal{M}_{i}$ and $N \in \mathcal{M}_{j}$. This together with the linearity of $\omega_{1}$ ensures that the topology is $T_{1}$.

To prove $X$ is monotonically normal it suffices [B] to define an open $H(x, W)$ for each $x \in X$ and neighborhood $W$ of $x$ such that:

(a) $x \in H(x, W) \subset W$.

(b) If $W \subset Z$, then $H(x, W) \subset H(x, Z)$.

(c) If $H(x, W) \cap H(y, Z) \neq \emptyset$, either $x \in Z$ or $y \in W$.

Such a function is called a monotone normality operator for $X$.

For each $x \in X$ and neighborhood $W$ of $x$, define $H(x, W)$ to be the union of all "basic open sets for $x$ " which are contained in $W$. Certainly (a) and (b) hold. Since $H(x, W)=\{x\}$ for $x \in\left(\omega_{1} \times \omega\right)$, to prove (c) it suffices to assume that $x=\langle\alpha, \omega\rangle, y=\langle\beta, \omega\rangle, \alpha<\beta<\omega_{1}, W$ is a neighborhood of $x$ and $Z$ a neighborhood of $y$. If $x \notin Z$, then $H(y, Z) \subset[((\beta+1)-(\alpha+1)) \times(\omega+1)]$. But $H(x, W) \subset[(\alpha+1) \times(\omega+1)]$. So $H(x, W) \cap H(y, Z)=\emptyset$ as desired and we have shown that $X$ is monotonically normal.

If $i \in \omega$, define $\mathcal{A}_{0}(i)=\left\{A \in \mathcal{A} \mid i \in I_{A 0}\right\}$ and $\mathcal{A}_{1}(i)=\left\{A \in \mathcal{A} \mid i \in I_{A 1}\right\}$.

Let $Y=(\omega+1)$ topologized as follows:

(1) If $M \in \mathcal{M}_{\omega}$ and $A \in \mathcal{A}, V_{M A}=\left(\{\omega\} \cup\left(I_{A 1} \cap M\right)\right)$ is a basic open set (for $\omega$ ) in $Y$.

(2) If $i \in \omega, M \in \mathcal{M}_{i}, A \in \mathcal{A}_{0}(i)$, and $B \in \mathcal{A}_{1}(i), V_{M A B}(i)=\left(I_{A 0} \cap I_{B 1} \cap M\right)$ is a basic open set (for $i$ ) in $Y$.

If $M$ and $M^{\prime}$ are both in $\mathcal{M}_{\omega}$ or $\mathcal{M}_{i}$, then $M \cap M^{\prime}$ is also. If $A$ and $A^{\prime}$ are in $\mathcal{A}_{0}(i),\left(A \cup A^{\prime}\right) \in \mathcal{A}_{0}(i)$ and $\left.\left(I_{A 0} \cap I_{A^{\prime} 0}\right)=I_{\left(A \cup A^{\prime}\right) 0}\right)$. One can replace 0 by 1 here. The basis defined by (1) and (2) is clearly a topology for $X$. Since for $i \neq j$ in $Y$ there are disjoint $M \in \mathcal{M}_{i}$ and $N \in \mathcal{M}_{j}$, the topology is Hausdorff. Also these 
separations as well as the separations by an $I_{\alpha 0}$ and $I_{\alpha 1}=\left(\omega-I_{\alpha 0}\right)$ yield disjoint open sets, so the topology is 0 -dimensional and regular.

It remains to prove that $(X \times Y)$ is not normal.

Define $F=\left\{\langle\langle\alpha, \omega\rangle, \omega\rangle \in(X \times Y) \mid \alpha \in \omega_{1}\right\}$. Since $\omega_{1} \times\{\omega\}$ is closed in $X$ and $\{\omega\}$ is closed in $Y, F$ is closed in $(X \times Y)$.

Define $K=\left\{\langle\langle\alpha, i\rangle, i\rangle \in(X \times Y) \mid \alpha \in \omega_{1}, i \in \omega\right\}$. We prove $K$ is closed by showing that $(X \times Y)-K$ is open.

If $\beta \in \omega_{1}$ and $M \in \mathcal{M}_{\omega}$, then $\langle\langle\beta, \omega\rangle, \omega\rangle \in\left(U_{M\{\beta\}(-1)}(\beta) \times V_{M\{\beta\}}\right)$ which does not intersect $K$ since no $i \in \omega$ can be in both $I_{\{\beta\} 0}$ and $I_{\{\beta\} 1}$.

Suppose $j \leq \omega, k \leq \omega$, and $j \neq k$. If $j=\omega$ there are disjoint $M \in \mathcal{M}_{\omega}$ and $N \in \mathcal{M}_{k}$ and $\langle\langle\beta, \omega\rangle, k\rangle \in\left(V_{M A(-1)}(\beta) \times V_{N B C}(k)\right)$ for arbitrary choices of $A \in \mathcal{A}_{\beta}, B \in \mathcal{A}_{0}(k)$, and $C \in \mathcal{A}_{1}(k)$, and this set misses $K$ since no $i \in \omega$ can be in both $M$ and $N$. If $j<\omega$ and $\beta \in \omega_{1},\langle\beta, j\rangle$ is isolated in $X$. There is $M \in \mathcal{M}_{k}$ not containing $j$ and $\langle\langle\beta, j\rangle, k\rangle \in(\{\langle\beta, j\rangle\} \times V)$ where $V=V_{M A}$ for some $A \in \mathcal{A}$ if $k=\omega$, or $V=V_{M B C}(k)$ for some choice of $B \in \mathcal{A}_{0}(k)$ and $C \in \mathcal{A}_{1}(k)$ if $k<\omega$. In either case $(\{\langle\beta, j\rangle\} \times V) \cap K=\emptyset$ since $j \notin V$.

Thus $F$ and $K$ are closed and disjoint. Assume that $G$ is an open set in $(X \times Y)$ containing $F$. We prove that $(X \times Y)$ is not normal by proving:

Claim. $\bar{G} \cap K \neq \emptyset$.

Proof of claim. For each $\alpha \in \omega_{1},\langle\langle\alpha, \omega\rangle, \omega\rangle \in G$. Thus there are basic neighborhoods $U_{\alpha}=U_{M_{\alpha} A_{\alpha} \lambda_{\alpha}}(\alpha)$ for $\langle\alpha, \omega\rangle$ in $X$ and $V_{\alpha}=V_{N_{\alpha} B_{\alpha}}$ for $\omega$ in $Y$ such that $\left(U_{\alpha} \times V_{\alpha}\right) \subset G$ and $\left(B_{\alpha}-A_{\alpha}\right) \neq \emptyset$.

Since $\left(M_{\alpha} \cap N_{\alpha}\right) \in \mathcal{M}_{\omega}$ which is countable, there is an $M \in \mathcal{M}_{\omega}$ and a stationary subset $S$ of $\omega_{1}$ such that $\left(M_{\alpha} \cap N_{\alpha}\right)=M \in \mathcal{M}_{\omega}$ for all $\alpha \in S$. For all $\alpha \in S$, $\langle\alpha, \omega\rangle \in U_{M A_{\alpha} \lambda_{\alpha}}(\alpha) \subset U_{\alpha}$ and $\omega \in V_{M B_{\alpha}} \subset V_{\alpha}$ so we can assume $M_{\alpha}=N_{\alpha}=M$ for all $\alpha \in S$.

Since $\lambda_{\alpha}<\alpha$ and $S$ is stationary, there is a $\lambda \in \omega_{1}$ and a stationary subset $S^{\prime}$ of $S$ such that $\lambda_{\alpha}=\lambda$ for all $\alpha \in S^{\prime}$.

For $i \in \omega$, let $S_{i}=\left\{\alpha \in S^{\prime} \mid i \in\left(I_{A_{\alpha} 0} \cap I_{\left(B_{\alpha}-A_{\alpha}\right) 1} \cap M\right)\right\}$.

Fix some $i$ for which $S_{i}$ is uncountable.

Let $\delta=\min S_{i}$. For all $\alpha \in S_{i}, \lambda<\delta \leq \alpha, A_{\alpha} \in \mathcal{A}_{\alpha}$, and $i \in\left(I_{A_{\alpha} 0} \cap M\right)$; so $\langle\delta, i\rangle \in U_{\alpha}$. If $j \in\left(I_{B_{\alpha} 1} \cap M\right)$ for some $\alpha \in S_{i}$, then $j \in V_{\alpha}$ and $\langle\langle\delta, i\rangle, j\rangle \in$ $\left(U_{\alpha} \times V_{\alpha}\right) \subset G$. Let $J=\left\{j \in \omega \mid j \in\left(I_{B_{\alpha} 1} \cap M\right)\right.$ for some $\left.\alpha \in S_{i}\right\}$. If $i \in$ (closure of $J$ in $Y$ ), then $\langle\langle\delta, i\rangle, i\rangle \in K \cap$ (closure of $G$ in $(X \times Y)$ ) proving our claim.

Otherwise there is a basic neighborhood $V_{N D E}(i)$ of $i$ in $Y$ not intersecting $J$. Since $i \in N,(N \cap M) \in \mathcal{M}_{i}$. Since for all $\alpha \in \omega_{1}, A_{\alpha} \in \mathcal{A}_{\alpha}, A_{\alpha} \subset\left(\omega_{1}-\alpha\right)$. Since $S_{i}$ is uncountable, there is some $\alpha \in S_{i}$ such that $A_{\alpha} \cap D=\emptyset$. Fix this $\alpha$. Since $i \in I_{\beta 1}$ for all $\beta \in\left(B_{\alpha}-A_{\alpha}\right)$ and $i \in I_{\gamma 0}$ for all $\gamma \in D,\left(B_{\alpha}-A_{\alpha}\right) \cap D=\emptyset$. Also $(E \cap D)=\emptyset$ since $i \in V_{N D E}(i)$.

Hence we can choose $j$ from the infinite set $\left[I_{D 0} \cap I_{\left(B_{\alpha} \cup E\right) 1} \cap(M \cap N)\right]$. But $j \in\left(V_{N D E}(i) \cap J\right)$ which contradicts our choice of $V_{N D E}(i)$.

Comment. We want to thank the referee for pointing out three interesting facts:

(One) In $[\mathrm{H}], \mathrm{R}$. W. Heath essentially proved that no countable dense subset of $2^{\omega_{1}}$ (with the usual product topology) is monotonically normal.

(Two) The countable space $Y$ constructed in the proof of Theorem 2 can be densely embedded in $2^{\omega_{1}}$. 
(Three) If $Y^{\prime}$ is any countable dense subset of $2^{\omega_{1}}$, there is a monotonically normal space $X^{\prime}$ such that $X^{\prime} \times Y^{\prime}$ is not normal.

Trivially, there is a natural homeomorphism $p:\left(2^{\omega} \times 2^{\omega_{1}}\right) \rightarrow 2^{\omega_{1}}$. Let $p_{1}$ : $\left(2^{\omega} \times 2^{\omega_{1}}\right) \rightarrow 2^{\omega}$ and $p_{2}:\left(2^{\omega} \times 2^{\omega_{1}}\right) \rightarrow 2^{\omega_{1}}$ be the projections.

Proof of (One). We may assume the $f_{n}$ 's defined in the proof of Theorem 2 satisfy $f_{n}(j)=1$ for all $j<n$. Define $g: Y(=\omega+1) \rightarrow\left(2^{\omega} \times 2^{\omega_{1}}\right)$ by having $k=p_{1} \circ g$ and $h=p_{2} \circ g$ where $k(\omega)(j)=1$ for all $j \in \omega$ and $h(\omega)(\alpha)=1$ for all $\alpha \in \omega_{1}$. For each $i \in \omega$, let $k(i) \in 2^{\omega}$ be the unique function such that $i \in M_{k(i)\lceil n}$ for each $n \in \omega$. Define $h \in 2^{\omega_{1}}$ by $h(i)(\alpha)=0$ if and only if $i \in I_{\alpha 0}$. It is then easy to check that $g$ is a dense embedding of $Y$ into $2^{\omega} \times 2^{\omega_{1}}$.

Proof of (Three). Let $Y^{\prime}$ be a countable dense subset of $2^{\omega} \times 2^{\omega_{1}}$. Since $y^{\prime}$ is countable we can assume that $Y^{\prime}=\omega+1$ as a set and $\omega$ is the point of $Y^{\prime}$ whose coordinates are all 1. For each $f \in 2^{<\omega}$, define $M_{f}=\left\{i \in \omega\left(=Y^{\prime}-\{\omega\}\right)\right.$ $p_{1}(i)$ is an extension of $\left.f\right\}$. Define $I_{\alpha 0}=\left\{i \in Y^{\prime}-\{\omega\} \mid p_{2}(\alpha)=0\right\}$. Again one easily checks that these $M_{f}$ 's and $I_{\alpha 0}$ 's operate with $Y^{\prime}$ exactly as the ones defined for $Y$ operated in the proof of Theorem 2.

Theorem 3. If $X$ is a monotonically normal space and $Y$ is a monotonically normal countable space, then $(X \times Y)$ is normal.

Proof. Using the standard proof that (regular) Lindelöf spaces are normal one can prove:

Lemma 3.1 ([S]). If $X$ is normal and $Y$ is countable, then $(X \times Y)$ is normal if and only if for all $y \in Y$ and closed $K$ in $(X \times Y)$ with $K \cap(X \times\{y\})=\emptyset,(X \times\{y\})$ and $K$ can be separated.

Fix such a $y$ and $K$. Then for the purposes of this paper we define $Z \subset X$ to be $o k$ if $Z$ is closed in $X$ and there is an open $O$ in $(X \times Y)$ with $(Z \times\{y\}) \subset O$ and $\bar{O} \cap K=\emptyset$. Our aim, of course, is to prove that $X$ is ok, thus by Lemma 3.1 proving Theorem 3.

Lemma 3.2. Every union of a closed discrete family of ok sets is ok.

Proof. Suppose $\mathcal{Z}=\left\{Z_{\alpha} \mid \alpha \in \kappa\right\}$ is a closed discrete family of ok sets and $Z=\bigcup \mathcal{Z}$. By definition $Z$ is closed and, for each $\alpha \in \kappa$, there is an open $U_{\alpha}$ in $X$ with $\left(Z_{\alpha} \times\{y\}\right) \subset U_{\alpha}$ while $\bar{U}_{\alpha} \cap K=\emptyset$. Since $X$ is monotonically normal and thus collectionwise normal, we can assume that $\left\{\bar{U}_{\alpha} \mid \alpha \in \kappa\right\}$ is closed discrete. Then $O=\bigcup\left\{U_{\alpha} \mid \alpha \in \kappa\right\}$ testifies to $Z$ being ok.

Lemma 3.3. Every closed subset of $X$ which is homeomorphic to a subset of an ordinal is ok.

Comment. This lemma is an obvious consequence of Theorem 1 for linearly ordered $X$ and countable $Y$. But for monotonically normal $X$ and countable $Y$, the lemma fails as the examples in Theorem 2 show. By adding monotone normality to countability for $Y$ we again have the lemma (and normality for $(X \times Y)$ ).

Proof of Lemma 3.3. If the lemma fails, there is a minimal ordinal $\kappa$ for which there is a non-ok closed $Z$ in $X$ homeomorphic to a subset of $\kappa$. We think of $Z$ as a subspace of both $X$ and $\kappa$ with its ordinary $\leq$ order topology. By Lemma 3.2 and the minimality of $\kappa, \kappa$ has uncountable cofinality and $Z$ is stationary in $\kappa$. 
For each $\alpha \in Z$ choose a basic open neighborhood $\left(U_{\alpha} \times V_{\alpha}\right)$ of $\langle\alpha, y\rangle$ such that $\left[\left(\bar{U}_{\alpha} \times \bar{V}_{\alpha}\right) \cap K\right]=\emptyset$ and let $\left\{y_{n} \mid n \in \omega\right\}$ be a one-to-one indexing of $(Y-\{y\})$.

Defining $Z^{*}$ for the GO space $Z$ as we defined $X^{*}$ for $X$ in Theorem 1 , let $p$ be the sup of $Z$ in $Z^{*}$. Then $p$ is a non- $Q$-gap point in $Z^{*}$. By Lemma $1.3,\langle p, y\rangle$ is not in the closure of $K \cap(Z \times Y)$ in $\left(Z^{*} \times Y\right)$ which implies that for some $\gamma \in Z$ and open neighborhood $V$ of $y$ in $Y,[(Z-\gamma) \times \bar{V}] \cap K=\emptyset$. Since $Z$ is the union of the two closed sets $(Z-\gamma)$ and $Z \cap(\gamma+1)$ and, by the minimality of $\kappa, Z \cap(\gamma+1)$ is ok, we can assume that $(Z \cap \gamma)=\emptyset$. Thus $(Z \times \bar{V}) \cap K=\emptyset$. Assume $V_{\alpha} \subset V$ for all $\alpha \in Z$.

Let $H$ and $J$ be monotone normality operators for $X$ and $Y$, respectively. (Such an operator is defined in the proof of Theorem 2.) Let $O=\bigcup\left\{H\left(\alpha, U_{\alpha}\right) \times\right.$ $\left.J\left(y, V_{\alpha}\right) \mid \alpha \in Z\right\}$.

Suppose $\langle x, w\rangle \in K$; then $w \neq y$.

If $w \notin \bar{V},[X \times(Y-\bar{V})]$ is an open neighborhood of $\langle x, w\rangle$ missing $O$.

Suppose $w \in \bar{V}$. Then $x \notin Z$ and we claim that in this case $[H(x, X-Z) \times$ $J(w, Y-\{y\})]$ misses $O$. If this claim is correct, $O$ is an open neighborhood of $(Z \times\{y\})$ in $(X \times Y)$ whose closure misses $K$. Thus $Z$ is ok and Lemma 3.3 holds.

So let us prove our claim by supposing that there is an $\alpha \in Z$ and $\langle p, q\rangle \in$ $\left([H(x, X-Z) \times J(w, Y-\{y\})] \cap\left[H\left(\alpha, U_{\alpha}\right) \times J\left(y, V_{\alpha}\right)\right]\right)$. Since $H$ is a monotonic normality operator on $X, \alpha \in Z$, and $p \in\left(H\left(\alpha, U_{\alpha}\right) \cap H(x, X-Z)\right), x \in U_{\alpha}$. Since $J$ is a monotonic normality operator on $Y$ and $q \in\left(J(w, Y-\{y\}) \cap J\left(y, V_{\alpha}\right)\right], w \in V_{\alpha}$. But we have a contradiction since $K \cap\left(U_{\alpha} \times V_{\alpha}\right)=\emptyset$ and $\langle x, w\rangle \in K$.

Lemma 3.4. $X$ is ok. (Which will complete the proof of Theorem 3.)

Since $K$ is closed in $(X \times Y)$ and misses $(X \times\{y\})$, for each $x \in X$ there is a basic neighborhood $\left(U_{x} \times V_{x}\right)$ of $\langle x, y\rangle$ whose closure misses $K$. Since $\mathcal{U}=\left\{U_{x} \mid x \in X\right\}$ is an open cover of the monotonically normal $X$, by Theorem (II) of [BR], there is a $\sigma$-disjoint partial refinement $\mathcal{W}$ of $\mathcal{U}$ such that $(X-\cup \mathcal{W})$ is the union of a closed discrete family $\mathcal{Z}$ of copies of subsets of ordinals.

By Lemma 3.3 each term of $\mathcal{Z}$ is ok, and by Lemma 3.2, $\bigcup \mathcal{Z}$ is ok. Let $O$ denote the resulting open set in $(X \times Y)$ containing $((\bigcup \mathcal{Z}) \times\{y\})$ with $\bar{O} \cap K=\emptyset$.

By definition $\mathcal{W}=\bigcup\left\{\left.\mathcal{W}_{n}\right|_{n \in \omega}\right\}$ where each $\mathcal{W}_{n}$ is a set of disjoint open sets each contained in a member of $\mathcal{U}$; let $W_{n}=\bigcup \mathcal{W}_{n}$. Also $(\bigcup \mathcal{Z}) \subset W_{\omega}=\{x \in X \mid\langle x, y\rangle \in$ $O\}$.

Since the monotonically normal $X$ is countably paracompact $[\mathrm{BR}]$, there is a locally finite refinement $\left\{G_{n} \mid n \leq \omega\right\}$ of $\left\{W_{n} \mid n \leq \omega\right\}$ with $G_{n} \subset W_{n}$ for all $n \leq \omega$.

Then $\mathcal{E}=\left\{\left(G_{n} \cap W\right) \mid n<\omega\right.$ and $\left.W \in \mathcal{W}_{n}\right\}$ is a locally finite open cover of $X-W_{\omega}$. For $E \in \mathcal{E}$, let $f(E)$ denote a point $x$ with $E \subset U_{x}$. For $x \in X$, let $E_{x}=\bigcup\{E \in \mathcal{E} \mid f(E)=x\}$.

Finally let $\mathcal{M}=\left\{E_{x} \times V_{x} \mid x \in X\right\}$. Recall that $E_{x} \subset U_{x}$ and $\left(\bar{U}_{x} \times \bar{V}_{x}\right) \cap K=\emptyset$. Since $\mathcal{E}$ is locally finite, $((\overline{\cup \mathcal{M}}) \cap K)=\emptyset$. Hence $(O \cup(\bigcup \mathcal{M}))$ is an open set in $(X \times Y)$ containing $(X \times\{y\})$ whose closure misses $K$, as required for Lemma 3.4 (and Theorem 3).

\section{REFERENCES}

[B] C. J. R. Borges, A study of monotonically normal spaces, Proc. Amer. Math. Soc. 38 (1973), 211-214. MR 48:2994

[BR] Z. Balogh and M. E. Rudin, Monotone normality, Topology Appl. 47 (1992), 115-127. MR 94b:54065 
[F] V. V. Fedorčuk, On ordered spaces, Dokl. Akad. Nauk. SSSR 169 (1966), 778-880; English Transl., Soviet Math. Dokl. 7 (1966), 1011-1014. MR 34:8375

[GH] L. Gillman and M. Henriksen, Concerning rings of continuous functions, Trans. Amer. Math. Soc. 77 (1954), 340-362. MR 16:156g

[H] R. W. Heath, An easier proof that a certain countable space need not be stratifiable, Proc. Washington State Univ. Conf. on General Topology, Pullman, Wash. (1970) 56-59. MR 42:1044

[M] K. Morita, On the product of paracompact spaces, Proc. Japan Acad. 39 (1963), 559-563. MR 28:2519

[P] S. Purisch, The orderability and closed images of scattered spaces, Trans. Amer. Math. Soc. 320 (1990), 713-725. MR 90k:54044

[S] M. Starbird, The normality of products with a compact or metric factor, Thesis, U. of Wis., Madison, (1974).

Department of Mathematics, Barry University, Miami Shores, Florida 33161

Department of Mathematics, University of Wisconsin, Madison, Wisconsin 53706 